\title{
A Specification-Based Coverage Metric to Evaluate Test Sets
}

\section{Paul E. Ammann}

George Mason University

Information \& Software Engineering Dept.

Fairfax, VA 22033

\section{Paul E. Black}

U.S. DEPARTMENT OF COMMERCE Technology Administration National Institute of Standards and Technology 100 Bureau Drive

Gaithersburg, MD 20899

GC 



\section{A Specification-Based Coverage Metric to Evaluate Test Sets}

\section{Paul E. Ammann}

George Mason University

Information \& Software Engineering Dept.

Fairfax, VA 22033

\section{Paul E. Black}

U.S. DEPARTMENT OF COMMERCE Technology Administration National Institute of Standards and Technology 100 Bureau Drive

Gaithersburg, MD 20899

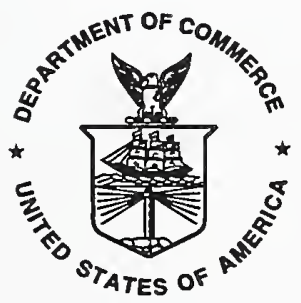

U.S. DEPARTMENT OF COMMERCE William M. Daley, Secretary

TECHNOLOGY ADMINISTRATION Gary R. Bachula, Acting Under Secretary for Technology

NATIONAL INSTITUTE OF STANDARDS AND TECHNOLOGY

Raymond G. Kammer, Director 



\title{
A Specification-Based Coverage Metric to Evaluate Test Sets
}

\author{
Paul E. Ammann* \\ George Mason University \\ pammann@gmu.edu
}

\author{
Paul E. Black \\ NIST \\ paul.black@nist.gov
}

\begin{abstract}
Software developers use a variety of methods, including both formal methods and testing, to argue that their systems are suitable for building high assurance applications. In this paper, we develop another connection between formal methods and testing by defining a specification-based coverage metric to evaluate test sets. Formal methods in the form of a model checker supply the necessary automation to make the metric practical. The metric gives the software developer assurance that a given test set is sufficiently sensitive to the structure of an application's specification. In this paper, we develop the necessary foundation for the metric and then illustrate the metric on an example.
\end{abstract}

\section{Introduction}

There is an increasing need for high quality software, particularly for highassurance applications such as avionics, medical, and other control systems. Developers have responded to this need in many ways, including improving the process, increasing the attention on early development activities, and using formal methods for describing requirements, specifications, and designs. Although all of these improvements contribute to better software, software still requires testing, and thus precise criteria are required to evaluate such

"Supported in part by the National Institute of Standards and Technology and in part by the National Science Foundation under grant CCR-99-01030. 
testing. In this paper we define one possible such criterion and explain how the criterion could be measured with respect to the software's specifications.

There are many approaches to generating tests $[3,7,15,20,24,29,30]$. There are also measures of the completeness, adequacy, or coverage of tests on source code [32]. However there are few objective measures of coverage that are independent of the implementation [12]. We have developed an innovative method that combines mutation analysis and model checking, which is useful for evaluating the coverage of system tests, comparing test generation methods, and minimizing test sets. Most coverage metrics apply to source code, which makes them difficult to apply in cases of conformance testing or developing tests before the code is finished. Since our method measures coverage over specifications, it can be used to evaluate test sets independent of code.

In this paper we categorize mutations of temporal logic formulae with respect to specification coverage analysis (Sect. 2.1). We then explain reflection, in which a state machine description is rewritten into a temporal logic (Sect. 2.2), and define expounding, in which implicit aspects of a model checking specification are made explicit (Sect. 2.3). We describe how to symbolically evaluate a test set for mutation adequacy (Sect. 2.4). Using the preceding techniques as a foundation, we define the specification coverage metric (Sect. 3). We illustrate these ideas with the Safety Injection example [8, 9] (Sect. 4).

\subsection{Background and Related Work}

Traditional program mutation analysis [14] is a code-based method for developing a test set that is sensitive to any small syntactic change to the structure of a program. A mutation analysis system defines a set of mutation operators. Each operator is a pattern for a small syntactic change. A mutant program, or more simply, mutant, is produced by applying a single mutation operator exactly once to the original program. The rationale is that if a test set can distinguish the original program from a mutant, the test set exercises that part of the program adequately. Applying the set of operators systematically generates a set of mutants. Some of these mutants may still be equivalent to the original program. A test set is mutation adequate if at least one test in the test set distinguishes each nonequivalent mutant. There are test data generation systems that, except for the ever-present undecidability problem, attempt to automatically generate mutation adequate test inputs [15]. Very little work on mutation analysis for specifications has been reported in the literature; however, Woodward did apply mutation 
analysis to algebraic specifications [30].

The example we use in this paper was originally coded using the Software Cost Reduction (SCR) method [19]. It is used to formally capture and document the requirements of a software system. It is scalable and its semantics are easy to understand; this accounts for the use of the SCR method and its derivatives in specifying practical systems [16, 18, 27]. Research in automated checking of SCR specifications includes consistency checking and model checking. The NRL SCR toolkit includes the consistency checker of Heitmeyer, Jeffords, and Labaw [17]. The checker analyzes application-independent properties such as syntax, type mismatches, missing cases, circular dependencies and so on, but not application-dependent properties such as safety and security. The toolkit also includes a backend translator to the model checker SPIN [21]. Atlee's model checking approach $[4,5,6]$ expresses an SCR mode transition table as a logic model and the safety properties as logic formulae and uses a model checker to determine if the formulae hold in the model. Owre, Rushby, and Shankar [26] describe how the model checker in PVS can be used to verify safety properties in SCR mode transition tables.

The model checking approach to formal methods specifies a system with a state transition relation and then characterizes the relation with properties stated in a temporal logic. Model checking has been successfully applied to a wide variety of practical problems, including hardware design, protocol analysis, operating systems, reactive systems, fault tolerance, and security. Although model checking began as a method for verifying hardware designs, there is growing evidence that it can be applied with considerable automation to specifications for relatively large software systems, such as the 'own-aircraft' logic for TCAS II [11]. Mutation analysis of specifications yields mutants from which the SMV model checker generates counterexamples that can be used as test cases [3]. The increasing utility of model checkers suggests using them in aspects of software development other than pure analysis, which is their primary role.

The chief advantage of model checking over the competing approach of theorem proving is complete automation. Human interaction is generally required to prove all but the most trivial theorems. Readily available model checkers such as SMV and SPIN can explore the state spaces for finite, but realistic, problems without human guidance [13]. We use the SMV model checker. It is freely available from Carnegie Mellon University and elsewhere. 


\section{Our Technology}

Our method begins with a specification of the system and a set of tests to be evaluated against the specification; see Figure 1. Although the specification need not be a complete description of the system, the more detailed the specification, the more that can be checked. We generate many variants of the original specification, or mutants. The set of tests are converted to finite state machines and are symbolically executed, one at a time. Some mutants are found to be inconsistent, that is, the model checker finds a difference between the symbolic execution of the test case and this mutant. Since we assume that the test cases are consistent with the original specification, this indicates an inconsistency with the original specification. If a mutant is found to be inconsistent by any of the test cases in the test set, it is considered to be killed by the test set. The ratio of killed mutants to total mutants is a coverage metric, similar to that of Wu et. al. [31], but applied to specifications. In general, the higher the ratio, the better or more completely the test set covers the specification. The lower the number, the less complete the covering.

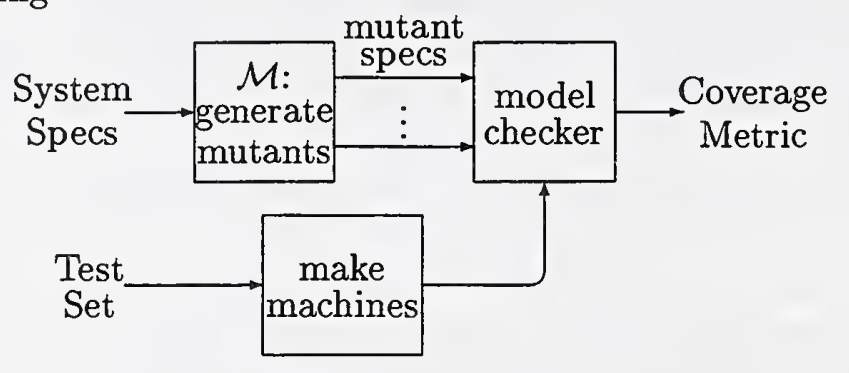

Figure 1: Specification coverage flow

Generally, testing is an attempt to assess the quality of a piece of software. If a test set inadequately exercises some part of the software, the assessment is less accurate. Since the software should correspond with the specification, a test set with better coverage of the specification is likely to more accurately assess the quality of a piece of software.

Program-based mutation analysis relies on the competent programmer hypothesis: programmers are likely to construct programs close to the correct program, and hence test data that distinguish syntactic variations of a given program are, in fact, useful. Here we assume an analogous "competent specifier hypothesis," which states that an analyst will write specifications which are likely to be close to what is desired. Hence test cases which distinguish syntactic variations of a specification are, in fact, useful. 


\subsection{Categories of Specification Mutations}

A specification for model checking has two parts. One is a state machine defined in terms of variables, initial values for the variables, and a description of conditions under which variables may change value. The other part is temporal logic constraints on valid execution paths. Conceptually, a model checker visits all reachable states and verifies that the invariants and temporal logic constraints are satisfied. Model checkers exploit clever ways of avoiding brute force exploration of the state space, for example, see [10].

Figure 2 illustrates the difference between mutations to logic formulae and mutations to program code. Code mutants are classified as either equivalent or nonequivalent. An equivalent mutant is one which has exactly the same input/output relation as the original program. ${ }^{1}$

Logic Mutants

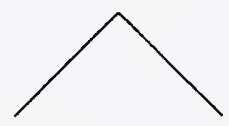

Consistent Inconsistent

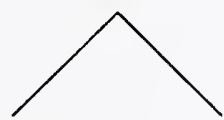

Falsifiable Nonfalsifiable
Code Mutants

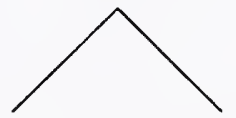

Equivalent Nonequivalent

Figure 2: Categories of mutants

Mutations to logic constraints in a model checking specification result in a different situation. Instead of being either equivalent or nonequivalent, mutants are either consistent or inconsistent with the state machine. A consistent mutant is simply a temporal logic formula that is true over all possible traces defined by the state machine. Just as equivalent mutants cannot be distinguished from the original for program-based mutation analysis, ${ }^{2}$ consistent mutants cannot be found false for model checking mutation analysis. Fortunately, consistency is decidable for these temporal logics, and model checkers are specifically designed to efficiently determine whether or

\footnotetext{
${ }^{1}$ We refer only to the "strong" version of program-based mutation analysis [14] here. In it a test case kills a mutant if execution reaches the mutant (the execution property [28]), the mutant corrupts the internal state (the infection property), and the corrupt internal state eventually results in an incorrect output (the propagation property). For weak mutation testing [22], only the execution and infection properties are required.

${ }^{2}$ For strong mutation testing, equivalent mutants compute the same input output pairs as the original program. Hence no test case can distinguish an equivalent mutant from the original program.
} 
not a temporal logic formula is consistent. So in this arena we do not have the problem of undecidability or requiring human judgement.

We evade the undecidability problem by working in the finite state space of the model checker. Not only is equivalent mutant identification possible in the context of a model checker, but model checkers are designed to perform this equivalency check efficiently. Therefore, equivalent mutants are not a problem for the specification-based mutation analysis which we present in this paper.

For inconsistent mutants, there are two possibilities. Some temporal logic formulae can be shown inconsistent with a single trace through the state machine. For example, if the assertion "the East-West light is never green while the North-South light is green" were inconsistent, the inconsistency could be exhibited with an execution trace that starts in some initial condition and ends in a state where both lights are green. We call mutants that are demonstrably inconsistent falsifiable. Other temporal logic formulae may be inconsistent with respect to the state machine, but cannot be shown inconsistent with a single trace. For example, an inconsistent assertion that "eventually both the East-South and West-North left turn lights are green simultaneously" cannot be shown to be false with any single trace from the state machine. We call mutants that are inconsistent but lack a counterexample nonfalsifiable.

\subsection{Expressing Specifications in CTL}

In our method, mutations are applied to temporal logic formulae. It is possible, and indeed desirable, to take advantage of existing constraints, such as safety assertions. However, such constraints may not be available, and, in any case, they are typically relatively loose constraints on the state machine. The difficulty with loose constraints is that mutants derived from them may be insensitive to many possible variations of the state machine.

To overcome this problem, we mechanically derive a set of temporal logic formulae for mutation. These formulae restate in temporal logic, or reflect, of the state machine's transition relation. Although the process is conceptually straight forward, there are subtle issues that require attention. To our knowledge, the literature does not have a comprehensive treatment of this topic for model checkers. Atlee and Buckley faced a similar problem and developed their own solution [5].

In SMV, there are two ways to specify a state machine transition relation: either procedurally via next statements in the ASSIGN section, or via constraints in the TRANS section. 
The interesting next statements are conditionals of the form:

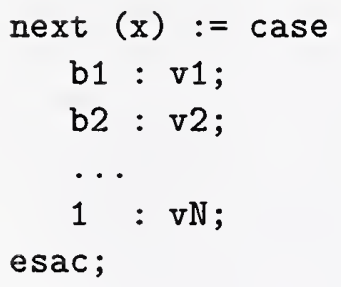

The semantics are typical of a programming language case statement. $b 1$ is evaluated; if it is true, $v 1$ is the next value for $x$. The right hand side, $v 1$ may be a set, thereby allowing for nondeterminism. If $b 1$ is false, b2 is evaluated. The case often ends in a default, which is 1, or true, in SMV.

To express the first case in CTL, one writes a formula such as:

$\operatorname{SPEC} A G(b 1 \rightarrow A X(x=v 1))$

This says that in all states (AG), if $b 1$ is true, all possible next states (AX) have $x=v 1$. If $v 1$ is a set, we write:

SPEC $A G(b 1 \rightarrow A X(x$ in $\mathrm{v} 1))$

For $b 2$, the situation is slightly more complicated. Preceding conditions, $b 1$ in this case, need to be subtracted out:

SPEC AG(!b1 \& b2 $\rightarrow A X(x=v 2))$

There are more subtle aspects to the process of determining the guards that we address in Sect. 2.3 below.

\subsubsection{Expressing next Clauses}

In the ASSIGN section, SMV allows the use of the next modifier for variable references; it evaluates the variable in the destination state instead of the current state. Unfortunately, the next modifier is not allowed in SPEC clauses. There are two routes out of this: create "shadow" variables that track the values from the previous state (this is the solution adopted in [5]), or access the variable after the $\mathrm{X}$ operator in CTL.

The first approach is simple, but it increases the number of variables, thereby potentially exploding the size of the state space. The second approach leads to a flat structure with potentially a large number of SPEC clauses. Consider the following next clause: 
next $(x):=$ case

$x=2 \& \operatorname{next}(y)=3: 5$;

...

esac;

Using the second approach, we refer to the value of $y$ in the next state.

SPEC AG( $\mathrm{x}=2 \rightarrow \operatorname{AX}(\mathrm{y}=3 \rightarrow \mathrm{x}=5))$

The potential for explosion arises when guards reference both current and next values of a variable.

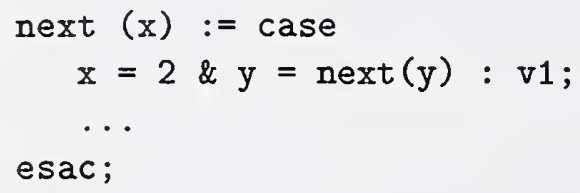

Using the second approach, we must explicitly enumerate the possible values for the variable, and test both before and after the $\mathrm{X}$ operator.

SPEC AG( $\mathrm{x}=2 \& \mathrm{y}=1 \rightarrow \operatorname{AX}(\mathrm{y}=1 \rightarrow \mathrm{x}=\mathrm{v} 1))$

$\operatorname{SPEC~} A G(x=2 \& y=2 \rightarrow \operatorname{AX}(y=2 \rightarrow x=v 1))$ ‥

If we use the first approach instead, we add a new variable, prevy, which keeps the previous value. The specification refers to previous values in future states.

next (prevy) := y;

SPEC AG( $\mathrm{x}=2 \rightarrow \mathrm{AX}($ prevy $=\mathrm{y} \rightarrow \mathrm{x}=\mathrm{v} 1))$

\subsubsection{Expressing TRANS Clauses}

For transition relations specified with the TRANS construct, reflection is simpler, since the TRANS constructs already are in CTL. Prefixing the predicate with the AG operator makes it a SPEC clause. The only issue is the use of the next operator, which can be handled in the same way as before, either with explicit "previous" values or judicious use of the $X$ operator. 


\subsubsection{Expressing Processes}

SMV also supports a process construct, whereby groups of changes to variables are gathered into modules. Process semantics are that one process is chosen at a time. The process construct conveniently mirrors the notion of an operation or transaction in traditional programming, including the notion of atomicity. The SPEC clauses of the temporal logic do not have an analogous structure associated with them. We suggest identifying explicitly the different processes and using the identifiers to write tight SPEC clauses for the reflection. So, changes to variables in process $p 1$ would be captured in the following template for a SPEC clause:

$\operatorname{SPEC} \operatorname{AG}(\ldots \rightarrow \operatorname{AX}(\operatorname{processID}=\mathrm{p} 1 \rightarrow \ldots))$

\subsection{Expounding}

As noted in the preceding section, the structure of guards must be elaborated when reflecting from the transition relations, since case statements have an implicit semantics based on syntactic order, whereas SPEC clauses are unordered. It turns out that for the purpose of mutation testing, more care is needed. In particular, it is easy to overspecify a SPEC clause. An overspecified clause yields a set of mutants that is not as sensitive as it could be.

An example may clarify matters. Consider the following statement from the Safety Injection problem:

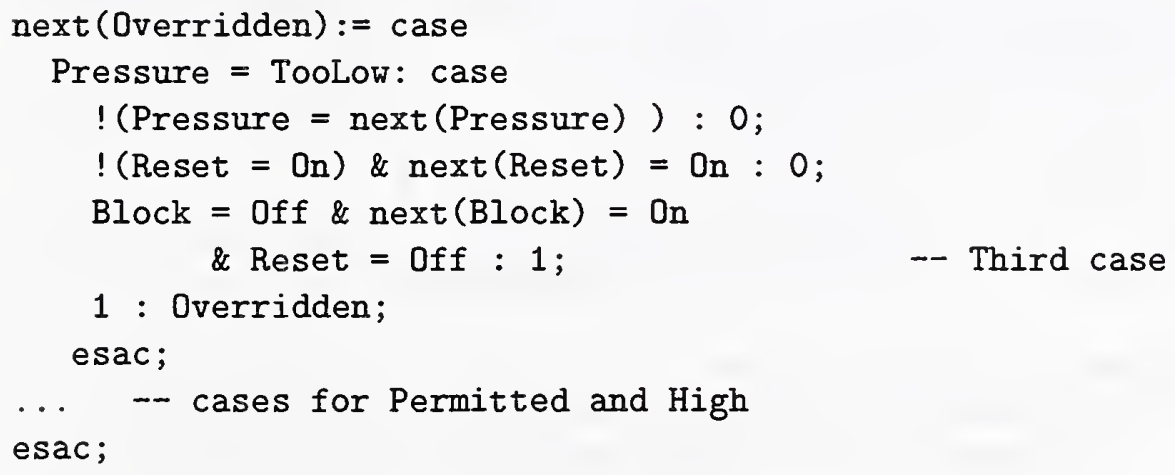

The third case, marked above, says that if Block is Off in the current state but is $O n$ in the next state and Reset is Off in the current state, Overridden is set to 1 (true) in the next state. Subtracting out the first two cases and simplifying would write the condition of the third case as: 


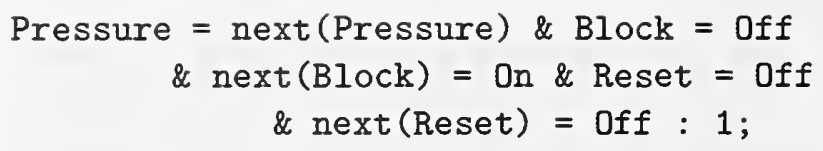

Notice that explicit consideration has been made for Pressure not changing, due to the first guard in the case statement, and Reset not changing, due to the second guard. This may be reflected in CTL as:

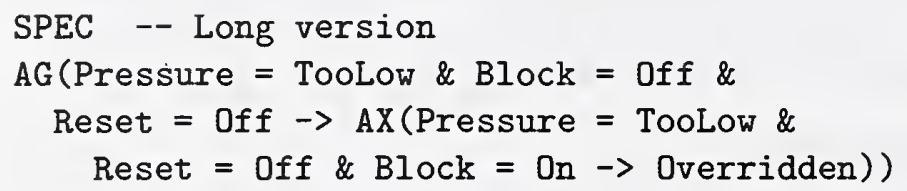

The following SPEC clause is also consistent because other parts of the specification constrain the way in which the variables may change. ${ }^{3}$

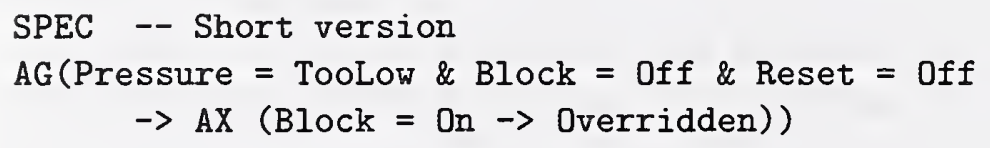

From a mutation analysis coverage metric perspective, this matters because a test set that kills all of the mutants generated from the long version does not necessarily kill all of the mutants generated from the short version. Consider what happens if a mutant operator changes the first occurrence of the predicate Pressure $=$ TooLow to Pressure $=$ Permitted. The two resulting SPEC clauses are as follows:

-- Mutation of long version

SPEC AG (Pressure = Permitted \& Block = Off \&

Reset $=$ Off $\rightarrow \mathrm{AX}$ (Pressure $=$ TooLow \&

Reset $=$ Off \& Block $=$ On $\rightarrow$ Overridden $)$ )

-- Mutation of short version

SPEC AG (Pressure = Permitted \& Block = Off \& Reset $=$ Off

$\rightarrow \mathrm{AX}$ (Block = On $\rightarrow$ Overridden))

The mutation of the long version is still consistent with respect to the state machine, because it is not possible for both Pressure and Block to

\footnotetext{
${ }^{3}$ For details, look at the TRANS specification of the complete example in the appendix. The relevant constraint is that only one of Pressure, Reset, and Block may change on any one transition.
} 
change on the same transition. Therefore, no valid test case can kill it. However, the short version mutation is both inconsistent and falsifiable.

How does one get enough redundancy to express the semantics of nonoverlapping alternatives of case statements, but not add redundancy that reduces with falsifiable mutants? Procedurally, it is quite simple: systematically drop predicates from the long version and run the model checker on the result. If the result is still consistent, the dropped predicate is redundant and can be omitted during mutation analysis.

An alternate strategy is to use the boolean derivative [1]. Consider a predicate $P$ that contains a boolean condition $x$. If $d P / d x$ evaluates to false, this implies that $P$ does not depend on $x$, and $x$ can safely be dropped from $P$.

As a procedural aside, we found it helpful to use Karnaugh maps to simplify the expressions resulting from expounding. We have not automated this aspect of specification preparation yet. We have found it to be relatively straight-forward using the model checker to examine the manual simplifications.

\subsection{Symbolic Execution of Test Cases}

Conceptually, a test case is a single trace through the state machine. We can express the test case as a constrained finite state machine, or CFSM, by adding a special variable, State, which controls the machine. Each original variable gets a new value depending solely on State. Otherwise it is unchanged.

Expressing a test case as a CFSM allows the model checker to symbolically execute the test case and check specifications for consistency. Consider the following simplified test case, which essentially turns Reset on then off again.

Reset $=$ Off; Block $=$ Off; Pressure $=$ TooLow;

STEP; Reset $=$ On; STEP; Reset = Off; STEP;

ASSIGN statements that execute this test case are the following. Since Block and Pressure don't change during the test, their next-state specifications are trivial. The value of Reset is driven solely by the State.

VAR

State : $0 . .2$;

ASSIGN 


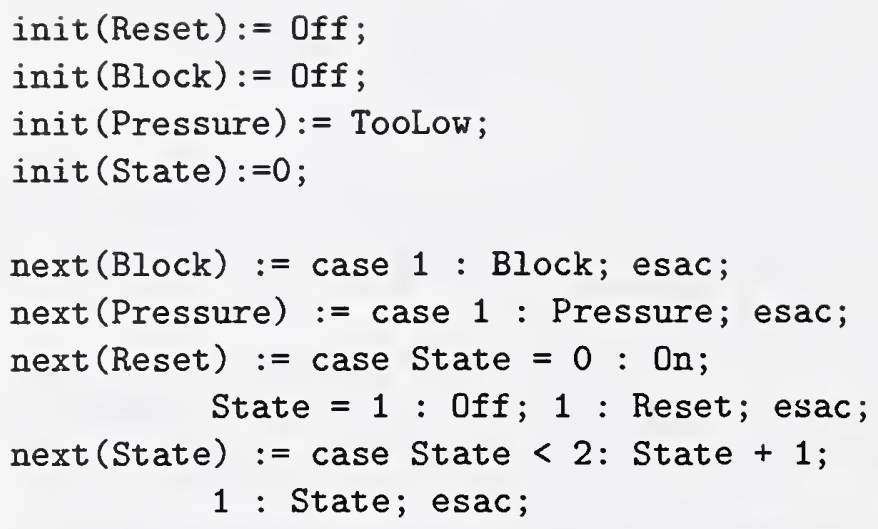

In a reactive system, such as Safety Injection, freezing the state at the end of the test is acceptable. However consider systems that have no quiescent state, such as a free-running counter. Consistent specifications may indicate that the state always changes. The specification is inconsistent with a CFSM generated as described, since the state freezes, but conceptually the specification is not wrong.

One may elaborate the CFSM with a special variable, Check, and set it false when the test ends. All the specifications may be automatically rewritten to include Check and evaluate to true when Check is false. This rewriting is detailed in [2].

\section{A Specification Coverage Metric}

The specification coverage metric for a test set, $t$, over a specification, $r$ (for "requirements"), is conceptually simple. It is similar to the test data adequacy of Wu et al. [31], but must be applied to specifications, not programs. Given a method, $\mathcal{M}$, for creating a set of mutants, the score, $S$, is the number of mutants killed by the test set, $k$, divided by the total number of mutants, $N$, produced by $\mathcal{M}$ on $r$.

$$
S(\mathcal{M}, r, t)=\frac{k}{N}
$$

When the method, specification, and test set parameters are understood, we omit them, thus $S=\frac{k}{N}$. We usually express the score as a percentage. The lowest, or worst, score is $0 \%$ when no mutants are killed. The highest, or best possible, score is $100 \%$ when all mutants are killed. 


\subsection{Preparing the Specification}

The method $\mathcal{M}$ for creating a set of mutants has three parts:

1. preparing the specification,

2. generating mutants, and

3. winnowing the mutants.

We use reflection, which is described in Sect. 2.2, to produce a fully explicit temporal logic description of the state machine's transition relation and any TRANS constraints. A fully explicit specification yields a more precise mutation analysis. We must shorten the resulting clauses as described in Sect. 2.3

As pointed out there, a test set may kill all falsifiable mutants from overly-specified clauses, but still not kill all falsifiable mutants of the shorter versions. Let $\mathcal{M}_{s}$ be a mutation process that shortens clauses before producing mutants, and $\mathcal{M}_{l}$ be a mutation process that uses the longer, overlyspecified clauses. Suppose a test set $t_{1}$, kills all mutants from $\mathcal{M}_{l}$, but not all those from $\mathcal{M}_{s}$, and another test set $t_{2}$, kills all mutants from both. The scores using $\mathcal{M}_{s}$ show the difference between the two test sets, while using $\mathcal{M}_{l}$ does not:

$$
\begin{aligned}
& S\left(\mathcal{M}_{l}, r, t_{1}\right)=S\left(\mathcal{M}_{l}, r, t_{2}\right) \\
& S\left(\mathcal{M}_{s}, r, t_{1}\right)<S\left(\mathcal{M}_{s}, r, t_{2}\right)
\end{aligned}
$$

We can see that mutation analysis on the shortened clauses, $\mathcal{M}_{s}$ in this case, is a more precise metric.

\subsection{Mutation Operators}

The heart of mutation analysis is generating mutants. Completely unconstrained changes would yield mostly syntactically incorrect mutants which are entirely meaningless, so a set of mutation operators is used. Each operator specifies a small syntactic change that is likely to be meaningful. For example, the "wrong variable" operator replaces a single occurrence of a variable with another variable of compatible type. The specification $a \wedge b$ might yield $c \wedge b$. The "wrong relational operator" mutation operator replaces any of $\langle, \leq,=, \neq, \geq$, or $>$ with one of the other five possibilities. The specification $a<b \wedge c$ might yield $a=b \wedge c$ by replacing $<$ with $=$.

Kuhn showed [23] that some operators subsume others. That is, any test set that kills all mutants of a subsuming operator also kills all mutants 
of the subsumed operator, while the opposite is not true. Thus if we use a subsuming operator, we need not use the subsumed operator. This lets us get the same precision with fewer mutants.

We could get maximum precision by using every conceivable operator that is not subsumed by another operator. However we believe a carefully chosen set of mutation operators will yield a fraction of the mutants, but still give us excellent precision. Research is underway to determine good sets of mutation operators for different conditions.

\subsection{Winnowing Mutants}

The first step in winnowing mutants is to discard those that are consistent with the specification. For instance, here is a clause from an automobile cruise control specification that says if the cruise control mode is Override and the ignition is turned off, the cruise control goes $O f f$ :

$$
\begin{array}{r}
\text { AG }(\text { CMode = Override } \rightarrow \text { AX (PIgnited \& } \\
\text { ! Ignited } \rightarrow \text { CMode }=0 f f))
\end{array}
$$

A "replace constant" mutation operator may change the conditioning mode to Cruise, as below, but that is still consistent since turning the ignition off in Cruise mode should turn the cruise control off.

AG (CMode = Cruise $\rightarrow \mathrm{AX}$ (PIgnited \&

$$
\text { ! Ignited } \rightarrow \text { CMode }=0 f f) \text { ) }
$$

Since consistent mutants are impossible to kill, leaving them makes it impossible for any test set to get $100 \%$. If the number of consistent mutants is some fraction, $\alpha$, of the number of inconsistent mutants, the score without consistent mutants, $S$, is proportionally reduced by $1 /(1+\alpha)$. Specifically, the score distorted with consistent mutants, $S^{\prime}$, has the total number of mutants increased by the number of consistent mutants, $N_{c}$. If $N_{c}=\alpha N$, then by Equation 1:

$$
S^{\prime}=\frac{k}{N+N_{c}}=\frac{k}{N+\alpha N}=\frac{1}{1+\alpha} \frac{k}{N}=\frac{1}{1+\alpha} S
$$

We find $\alpha$ to be about $50 \%$, so scores would be reduced by about a third. Consistent mutants are easily detected by comparing them with the original machine language specification in a single run of the model checker.

The next step in winnowing would be to eliminate "false" mutants, that is, mutants that evaluate to false in all conditions. For instance, a "replace 
variable" operator might change AG ( $P$ \& $! Q$ ) into AG (Q \& !Q). Since false mutants are killed by any test, leaving false mutants inflates the score. If the number of false mutants is some fraction, $\beta$, of the number of non-false inconsistent mutants, the score is increased by $\frac{\beta}{1+\beta}(1-S)$, where $S$ is the score without false mutants. More formally, the new score, $S^{\prime}$, has both the number of killed mutants and the total number of mutants increased by the number of false mutants, $N_{f}$. If $N_{f}=\beta N$, then by Equation 1:

$$
\begin{aligned}
S^{\prime} & =\frac{k+N_{f}}{N+N_{f}}=\frac{k+\beta N}{N+\beta N}=\frac{k+\beta N+\beta k-\beta k}{(1+\beta) N} \\
& =\frac{(1+\beta) k+\beta(N-k)}{(1+\beta) N} \\
& =\frac{(1+\beta) k}{(1+\beta) N}+\frac{\beta(N-k)}{(1+\beta) N} \\
& =\frac{k}{N}+\frac{\beta}{1+\beta} \frac{N-k}{N} \\
& =\frac{k}{N}+\frac{\beta}{1+\beta}\left(1-\frac{k}{N}\right) \\
& =S+\frac{\beta}{1+\beta}(1-S)
\end{aligned}
$$

We find $\beta$ is about $7 \%$, so a score of $80 \%$ is increased to $81 \%$. Even a score of $0 \%$ is only increased to $6 \%$. False mutants may be detected by comparing all mutants and their negations with the original machine specification in a single run of the model checker. If AG (p) is always false, the negation, AG (!p), is always true. Thus a pair where AG (p) is inconsistent and $A G(! p)$ is consistent indicates that $A G(p)$ is false. Alternatively, a satisfaction checker can directly determine if a specification is false. Since the number of false mutants is low, we don't do this.

Another step in winnowing could be to eliminate semantic duplicates, that is, mutants that evaluate the same for all possible tests. For instance, suppose "negate expression" and "replace operator" are applied to AG $(P<Q)$ and produce $A G(! P<Q)$ and $A G(P>=Q)$ respectively. These are exactly the same: any test either kills both or neither. Leaving duplicate mutants, instead of removing all but one copy, adds more weight to the duplicates. In the extreme, suppose we generate 100 mutants, but one has 200 copies. A test set that kills the 99 unduplicated mutants but doesn't kill the duplicated mutant gets a score of $99 / 300=33 \%$. But a test set that only kills the duplicates gets a score of $201 / 300=67 \%$ ! 
Duplicate mutants may be detected by essentially comparing every mutant clause against every other mutant. For instance, if we have mutants $A G \mathrm{~m} 1, \mathrm{AG} \mathrm{m} 2$ and $\mathrm{AG} \mathrm{m} 3$, check the following.

$\mathrm{AG} \mathrm{m} 1=\mathrm{m} 2$

$\mathrm{AG} \mathrm{m} 1=\mathrm{m} 3$

$\mathrm{AG} \mathrm{m} 2=\mathrm{m} 3$

Only duplicates will be consistent. In practice, we can reduce the number of comparisons by running a few tests to quickly determine mutants that are not duplicates, then comparing possibly-duplicate mutants with each other. We have not yet characterized the number or distribution of duplicate mutants.

\begin{tabular}{|c|c|c|}
\hline Current Mode & Event & New Mode \\
\hline TooLow & $@ T($ WaterPres $\geq$ Low $)$ & Permitted \\
\hline Permitted & $@ T($ WaterPres $\geq$ Permit $)$ & High \\
\hline Permitted & $@ T($ WaterPres $<$ Low $)$ & TooLow \\
\hline High & $@ T($ WaterPres $<$ Permit $)$ & Permitted \\
\hline
\end{tabular}

Initial State : Mode $=$ TooLow, WaterPres $<$ Low

Mode transition table for Pressure.

\begin{tabular}{|c|c|c|}
\hline Mode & \multicolumn{2}{|c|}{ Events } \\
\hline High & False & $@ T($ Inmode $)$ \\
\hline TooLow & $@ T($ Block $=$ On $)$ & $@ T($ Inmode $)$ OR \\
Permitted & WHEN $($ Reset $=$ Off $)$ & $@ T($ Reset $=$ On $)$ \\
\hline \hline Overridden & True & False \\
\hline
\end{tabular}

Event table for Overridden.

\begin{tabular}{|c|c|c|}
\hline Mode & \multicolumn{2}{|c|}{ Conditions } \\
\hline High, Permitted & True & False \\
\hline TooLov & Overridden & NOT Overridden \\
\hline \hline Safety Injection & Off & On \\
\hline
\end{tabular}

Condition table for Safety Injection.

Table 1: Safety injection tables

\section{Example}

We applied our method to the Safety Injection problem. See App. A for the complete specification for SMV. Table 1 is a higher-level, tabular specification. We used three progressively more elaborate preparation methods: the reflected specification, the reflected specification with expounding, and 
the reflected specification with expounding and TRANS clauses. The expounded clauses were also shortened. The appendix shows the specification resulting from the third, most elaborate method. The specification resulting from the first method may be obtained from that in the appendix by dropping all the SPEC clauses tagged with an " $\mathrm{e}$ " or reflected from the TRANS relation.

Using the method in [3] we automatically generated test sets from all three versions of the specification. For comparison, we also manually produced a minimal test set that covered the tables expressed in Table 1. We explain the notion of "table coverage" below.

\subsection{Mutation Generation}

We used Vadim Okun's mutation engine with the following operators. We illustrate each operator with a mutant it generates from the following clause. Changes are emphasized by underlining.

$$
\begin{aligned}
& \text { AG (Pressure }=\text { TooLow \& Reset }=\text { Off } \rightarrow \\
& \mathrm{AX} \text { (Reset }=\text { On } \rightarrow \text { !Overridden)) }
\end{aligned}
$$

1. replace_constant: replace one constant with another, e.g.,

$$
\begin{aligned}
& \text { AG (Pressure = High \& Reset }=\text { Off } \rightarrow \\
& \mathrm{AX} \text { (Reset }=\text { On } \rightarrow \text { ! Overridden)) }
\end{aligned}
$$

2. replace_oper: replace one operator with another operator, e.g., replace "and" with "or"

$$
\begin{aligned}
& \text { AG (Pressure }=\text { TooLow } 1 \text { Reset }=\text { Off } \rightarrow \\
& A X \text { (Reset }=\text { On } \rightarrow \text { !Overridden)) }
\end{aligned}
$$

3. replace_vars: replace a variable with another variable, e.g.,

$$
\begin{aligned}
\text { AG (Pressure }=\text { TooLow \& } \underline{\text { Block }}=\text { Off } \rightarrow \\
\text { AX (Reset }=\text { On } \rightarrow \text { ! Overridden)) }
\end{aligned}
$$

4. remove_expr: remove a simple expression from conjunctions, disjunctions, and implications, e.g.,

$$
\begin{gathered}
\text { AG (Pressure }=\text { TooLow }-> \\
\text { AX (Reset }=\text { On } \rightarrow \text { ! Overridden)) }
\end{gathered}
$$




\begin{tabular}{|l|c|c|c|}
\hline & No Expound & $\begin{array}{c}\text { Expound } \\
\text { no TRANS }\end{array}$ & $\begin{array}{c}\text { Expound } \\
\text { with TRANS }\end{array}$ \\
\hline Mutant SPEC clauses & 188 & 611 & 1131 \\
Inconsistent SPEC mutants & 121 & 388 & 824 \\
\hline Test cases & 16 & 27 & 36 \\
Test cases after minimizing & 10 & 16 & 18 \\
\hline
\end{tabular}

Table 2: Mutations before and after expounding

The only winnowing we do is to exclude consistent mutants. Table 2 shows the results of applying mutation generation before expounding, after expounding without reflecting the TRANS clause, and after expounding and reflecting the TRANS clause. The table also shows the number of test cases automatically generated by the method in [3] for each version.

We can use our mutation analysis method to minimize test sets. We analyzed the mutants killed by different test cases and found a smaller set which has the same coverage. The last row of the table shows the number of test cases after this minimization. Interestingly, even though the number of mutants grows enormously with expounding and consideration of the TRANS clause, the number of test cases grows modestly.

\subsection{Evaluation of Separately Developed Test Sets}

Consider the SCR tables, shown in Table 1 , that gave rise to the SMV model. These tables are reproduced from [9]. Suppose we construct a test set that satisfies the following criteria: each row in a mode transition table is covered by one or more test cases. In addition, for each mode, the possibility of remaining in that mode is covered by one or more test cases. In an event table, the conditions that cause each event are forced to both true and false, if possible, on one or more test cases. Finally, in a condition table, each condition is forced to both true and false, if possible, on at least one test case. We call this metric table coverage. We define any test set that satisfies these criteria to be table adequate.

We produced a test set that satisfied the criteria for table coverage. ${ }^{5} \mathrm{We}$

\footnotetext{
${ }^{4}$ We found that for this example, all inconsistent mutants are falsifiable.

${ }^{5} \mathrm{SMV}$ can be used to check for table adequacy as follows. For each event in the transition table, a SPEC clause was written stating that the desired transition did not happen when the specified event occurred. SMV then generated a counterexample showing that the desired transition did happen if the event occurred. A similar strategy was applied to the event and condition tables. The result was 8 SPEC clauses for the mode transition table ( 2 for each row), 6 for the event table ( 2 for each event except False), and 2 for the condition table (all values for Overridden in mode TooLow), for a total of 16 SPEC
} 


\begin{tabular}{|lc|c|c|c|c|}
\hline & tests & $\begin{array}{c}\text { table coverage } \\
16 \text { elements }\end{array}$ & $\begin{array}{c}\text { No Expound } \\
121 \text { mutants }\end{array}$ & $\begin{array}{c}\text { Expound } \\
\text { no TRANS } \\
388 \text { mutants }\end{array}$ & $\begin{array}{c}\text { Expound } \\
\text { with TRANS } \\
824 \text { mutants }\end{array}$ \\
\hline table adequate & 4 & $100 \%(16)$ & $89 \%(108)$ & $77 \%(301)$ & $70 \%(577)$ \\
\hline No Expound & 16 & $100 \%(16)$ & $100 \%(121)$ & $81 \%(317)$ & $76 \%(629)$ \\
\hline $\begin{array}{c}\text { Expound } \\
\text { no TRANS }\end{array}$ & 27 & $100 \% \dagger$ & $100 \% \dagger$ & $100 \%(388)$ & $93 \%(767)$ \\
\hline $\begin{array}{c}\text { Expound } \\
\text { with TRANS }\end{array}$ & 36 & $100 \% \dagger$ & $100 \% \dagger$ & $100 \% \dagger$ & $100 \%(824)$ \\
\hline
\end{tabular}

Table 3: Test set coverage scores with different criteria

build a table adequate test set by picking a subset of the test cases generated for the unexpounded version of the safety injection problem. This turned out to be sufficient; otherwise, we could have produced additional tests. The result was a set of four test cases.

Table 3 shows the result of evaluating the table adequate test set and the three automatically generated test sets against the table coverage metric and also against our metric with mutant generation with no expounding, expounding without TRANS, and expounding with TRANS. The scores marked with $\dagger$ are derived rather than measured. We justify the derived scores because the mutants that define adequacy for the second test set are a subset of those that define the third, and the mutants that define adequacy for the third test set are a subset of those that define the fourth. The 16 tests generated from No Expound scored $317 / 388$ or $81 \%$ on mutants from Expound, but no reflection of TRANS clauses. Similarly, the 27 tests generated from Expound, No TRANS scored $767 / 824$ or $93 \%$ on mutants from Expound with TRANS. Therefore, the additional mutants from reflection of the TRANS clause and expounding result in a more precise metric.

Although we would not expect table coverage testing of the SCR table to be as thorough as the mutation scheme used in this paper, it is still instructive to evaluate such tests with respect to mutation adequacy. It demonstrates how our method can be used to evaluate test sets developed by other means. This is important because most existing software systems have large regression test sets associated with them, and it is very useful to analyze such test sets for gaps and redundancy. In the former case, additional tests can be added. In the latter case, redundant tests can be analyzed for removal.

All the test sets generated from mutation analysis turn out to be table

clauses. That is, a table adequate test set for the tables in Table 1 must satisfy these 16 elements. 
coverage adequate. However, we note that a significant number of mutants are not necessarily detected by the table coverage adequate test set. This suggests that our specification-based mutation adequacy coverage metric has practical utility.

\section{Summary and Conclusion}

Testing, particularly system testing, consumes a significant portion of the budget for software development projects. Formal methods, typically used in the specification and analysis phases of software development, offer an opportunity not only to reduce the cost of testing, but to increase confidence in the software through formal metrics for test thoroughness. We pursued this path by applying model checking and mutation analysis to the problem of test set coverage. The resulting coverage metric can be used independently of source code, and is appropriate for "black box" testing.

From an assurance perspective, we would like to say that a test set guarantees some property for the software, but, with some exceptions, this goal is beyond the limits of what testing can show. Instead, test metrics are developed to capture desirable properties of a test set. Most of the testing metrics available in the literature are defined at the unit or source code level. We balance this bias by offering a metric at the software system level.

In this paper, we developed a metric for evaluating test sets against state transition specifications in the context of model checker. The metric is based on mutation analysis. Mutation adequate test sets are sensitive to the precise structure of the artifact from which the mutations are generated, which in this case is a model checking specification. We developed the necessary foundation for defining the mutation metric, including the roles of reflection, expounding, mutation operators, and winnowing procedures. We showed how to take a set of externally developed test cases, turn each test case into a constrained finite state machine, and score the set against the metric.

Scalability is a concern for all realistic software engineering techniques. The scalability of our technique depends in part on how well model checkers can handle large software specifications. The successes of SPIN [21] and SMV [11] suggest that a specification-based test coverage metric may apply to a broad variety of software systems.

To evaluate scalability, we plan to apply this technique to a much larger and richer specification, namely the generic Flight Guidance System developed by Rockwell Collins for the academic community and available in a 
variety of forms [25]. We also plan to explore starting with higher level specifications, say in Z, UML, or operational semantics, and automatically generating model checker specifications. Additionally, we will devise other mutation operators and determine which set of mutation operators give the best coverage with the smallest set of tests. At the same time, we will theoretically and experimentally investigate the impact of duplicate mutants on our metric.

\section{Acknowledgments}

It is our pleasure to thank Angelo Gargantini and Connie Heitmeyer of the Naval Research Laboratory for supplying the SMV specifications for Safety Injection and for many helpful exchanges of data and ideas. We also thank Vadim Okun of the University of Maryland, Baltimore County, for the use of his mutation engine mute.

\section{References}

[1] S.B. Akers. On a theory of boolean functions. SIAM Journal, 7(4), 1959.

[2] Paul Ammann and Paul E. Black. Abstracting formal specifications to generate software tests via model checking. In Proceedings of the 18th Digital Avionics Systems Conference (DASC99), to be published 1999.

[3] Paul E. Ammann, Paul E. Black, and William Majurski. Using model checking to generate tests from specifications. In Proceedings of the Second IEEE International Conference on Formal Engineering Methods (ICFEM'98), pages 46-54. IEEE Computer Society, December 1998.

[4] Joanne M. Atlee. Native model-checking of SCR requirements. In Proceedings of the Fourth International SCR Workshop, November 1994.

[5] Joanne M. Atlee and M. A. Buckley. A logic-model semantics for SCR software requirements. In Proceedings of the 1996 International Symposium on Software Testing and Analysis, pages 280-292, January 1996.

[6] Joanne M. Atlee and J. Gannon. State-based model checking of eventdriven system requirements. IEEE Transactions on Software Engineering, 19(1):24-40, January 1993. 
[7] Jonathan Beskin. Human error simulation in test case generation. Technical report, Reliable Software Technologies Corp., Sterling, Virginia, USA, 20166, 1998.

[8] Ramesh Bharadwaj and Constance Heitmeyer. Verifying SCR requirements specifications using state exploration. In Proceedings of the First ACM SIGPLAN Workshop on Automatic Analysis of Software, Paris, France, January 1997.

[9] Ramesh Bharadwaj and Constance L. Heitmeyer. Model checking complete requirements specifications using abstraction. Technical Report NRL/MR/5540- 97-7999, U.S. Naval Research Laboratory, November 1997.

[10] Jerry R. Burch, Edmund Melson Clarke, Jr., Ken L. McMillan, David L. Dill, and L. J. Hwang. Symbolic model checking: $10^{20}$ states and beyond. In Proceedings of the ACM/SIGDA International Workshop in Formal Methods in VLSI Design. ACM, January 1991.

[11] William Chan, Richard J. Anderson, Paul Beame, Steve Burns, Francesmary Modugno, David Notkin, and Jon D. Reese. Model checking large software specifications. IEEE Transactions on Software Engineering, 24(7):498 - 520, July 1998.

[12] Tsun S. Chow. Testing software design modeled by finite-state machines. IEEE Transactions on Software Engineering, SE-4(3):178-187, May 1978.

[13] Edmund Melson Clarke, Jr., Orna Grumberg, and David E. Long. Verification tools for finite-state concurrent systems. In A Decade of Concurrency - Reflections and Perspectives. Springer Verlag, 1994. Lecture Notes in Computer Science 803.

[14] Richard A. De Millo, Richard J. Lipton, and Frederick G. Sayward. Hints on test data selection: Help for the practicing programmer. IEEE Computer, 11(4):34-41, April 1978.

[15] Richard A. De Millo and A. Jefferson Offutt. Constraint-based automatic test data generation. IEEE Transactions on Software Engineering, 17(9):900-910, September 1991.

[16] S. R. Faulk, L. Finneran, J. Kirby Jr., S. Shah, and J. Sutton. Experience applying the CoRE method to the Lockheed C-130J. In Proceed- 
ings of the 9th Annual Conference on Computer Assurance, pages 3-8, Gaithersburg, MD, June 1994.

[17] Constance L. Heitmeyer, R. D. Jeffords, and B. G. Labaw. Automated consistency checking of requirements specifications. ACM Transactions on Software Engineering and Methodology, 5(3):231-261, July 1996.

[18] Constance L. Heitmeyer and J. Mclean. Abstract requirements specifications: A new approach and its application. IEEE Transactions on Software Engineering, SE-9(5):580-589, September 1983.

[19] K. L. Heninger. Specifying software requirements for complex systems. IEEE Transactions on Software Engineering, SE-6(1):2-13, January 1980.

[20] Daniel Hoffman, Paul Strooper, and Lee White. Boundary values and automated component testing. Software Testing, Verification $\mathcal{B}$ Reliability, 9(1), March 1999.

[21] Gerald J. Holzmann. The model checker SPIN. IEEE Transactions on Software Engineering, 23(5):279-295, May 1997.

[22] W. E. Howden. Weak mutation testing and completeness of test sets. IEEE Transactions on Software Engineering, 8(4):371-379, July 1982.

[23] D. Richard Kuhn. Fault classes and error detection in specification based testing. ACM Transactions on Software Engineering Methodology, to be published 1999.

[24] Peter M. Maurer. Generating test data with enhanced context-free grammars. IEEE Software, pages 50-55, July 1990.

[25] S. P. Miller. Specifying the mode logic of a flight guidance system in CoRE and SCR. In Second Workshop on Formal Methods in Software Practice, Clearwater Beach, FL, March 1998.

[26] Sam Owre, John M. Rushby, and N. Shankar. Analyzing tabular and state-transition requirements specifications in PVS. Technical Report CSL-95-12, Computer Science Laboratory SRI International, June 1995. Revised April, 1996.

[27] A. J. van Schouwen, D. L. Parnas, and J. Madey. Documentation of requirements for computer systems. In Proceedings of the IEEE International Symposium on Requirements Engineering, pages 198-207, San Diego, CA, January 1993. IEEE Computer Society Press. 
[28] Jeffrey M. Voas. PIE: A dynamic failure-based technique. IEEE Transactions on Software Engineering, 18(8), August 1992.

[29] Elaine Weyuker, Tarak Goradia, and Ashutosh Singh. Automatically generating test data from a boolean specification. IEEE Transactions on Software Engineering, 20(5):353-363, May 1994.

[30] Martin R. Woodward. Errors in algebraic specifications and an experimental mutation testing tool. Software Engineering Journal, pages 211-224, July 1993.

[31] D. Wu, M. A. Hennell, D. Hedley, and I.J. Riddell. A practical method for software quality control via program mutation. In Proceedings of the 2nd Workshop on Software Testing, Verification and Analysis, pages 159-170, Banff, Canada, July 1988.

[32] Hong Zhu, Patrick A. V. Hall, and John H. R. May. Software unit test coverage and adequacy. ACM Computing Surveys, 29(4):366-427, December 1997.

\section{A Safety Injection Problem}

The specification given below is a modification of one supplied by the Navy Research Laboratory. The specification corresponds to Table 1. See [9] for a closely related specification in all of SCR, SPIN, and SMV.

The numerical comments to the right of case statement branches below provide a cross reference between the transition relation and the reflection into SPEC clauses. Branches marked with an "e" require expounding prior to reflection. After expounding, it is often convenient to use multiple SPEC clauses for reflection; hence the multiple SPEC clauses for each of the "e" branches. In this example, only the default cases require expounding.

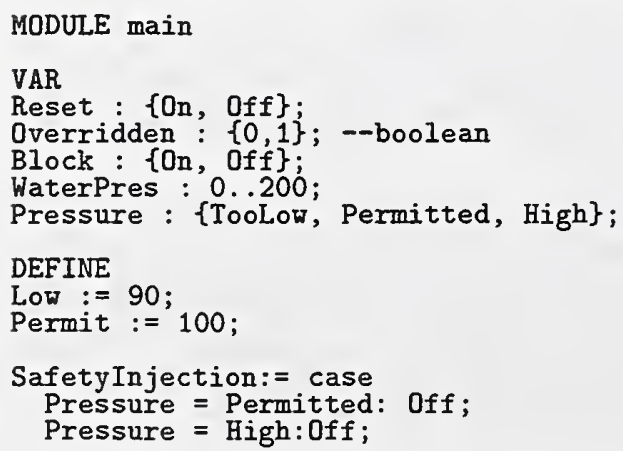




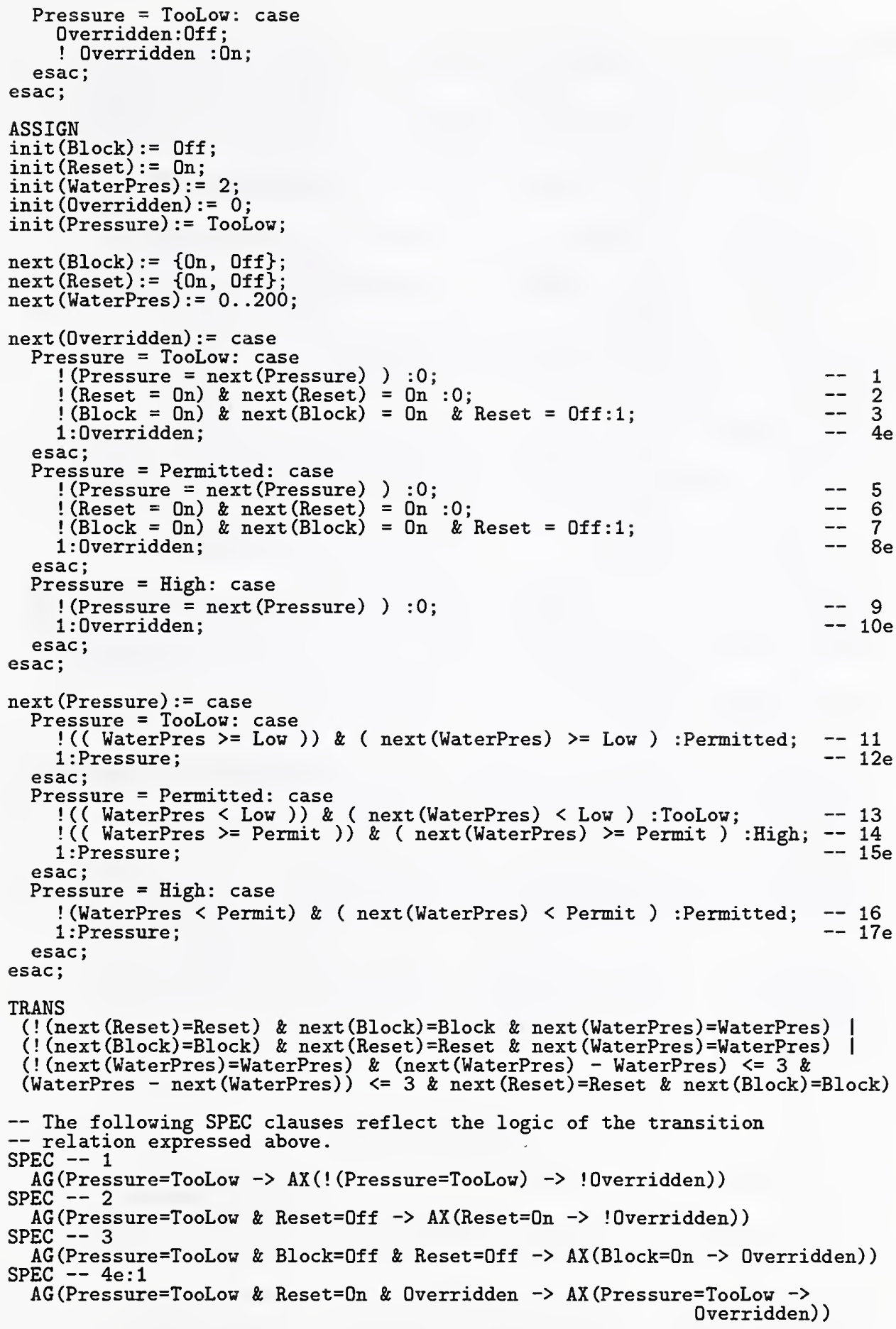




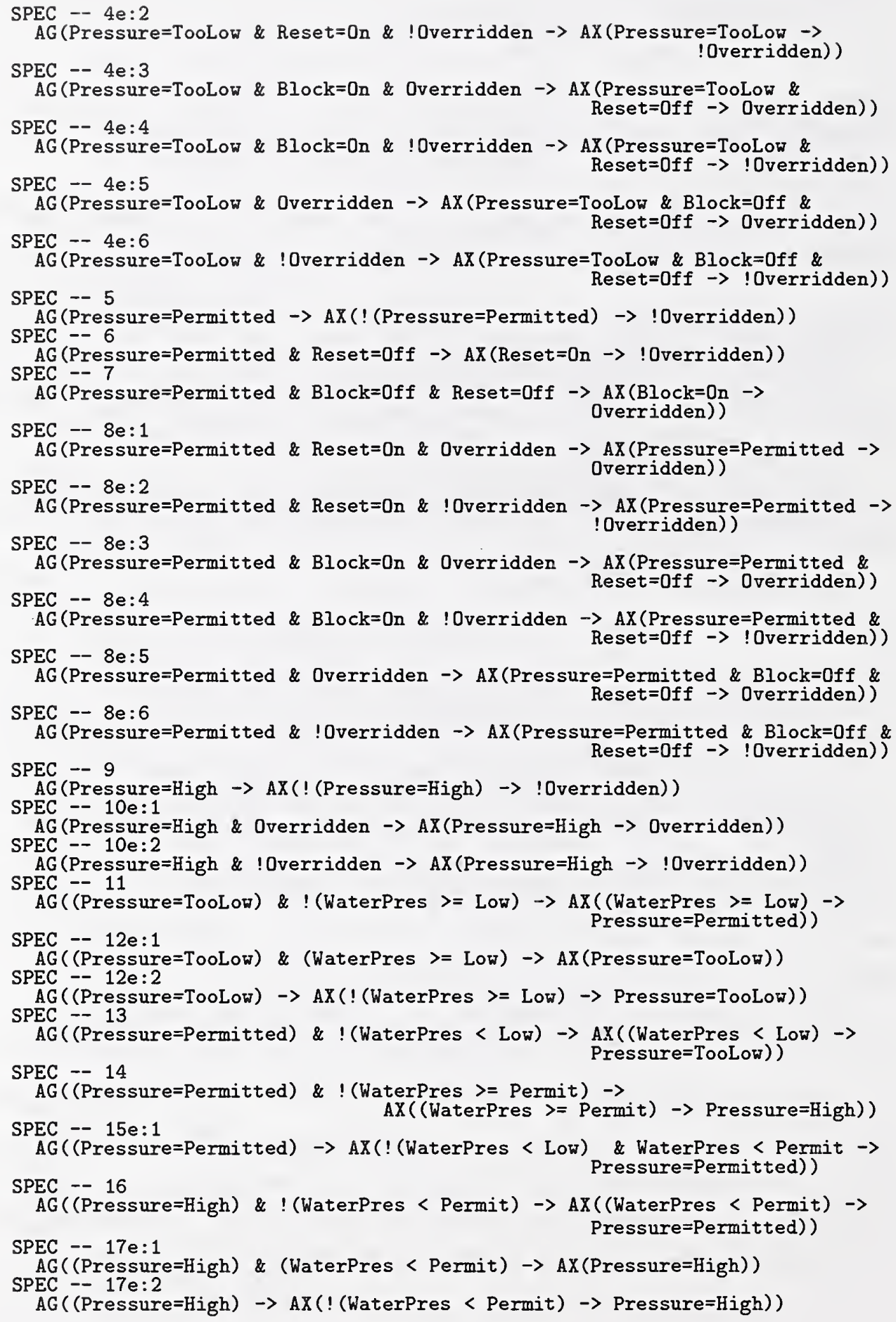


-- The following SPEC clauses reflect (an abstraction of) the TRANS section

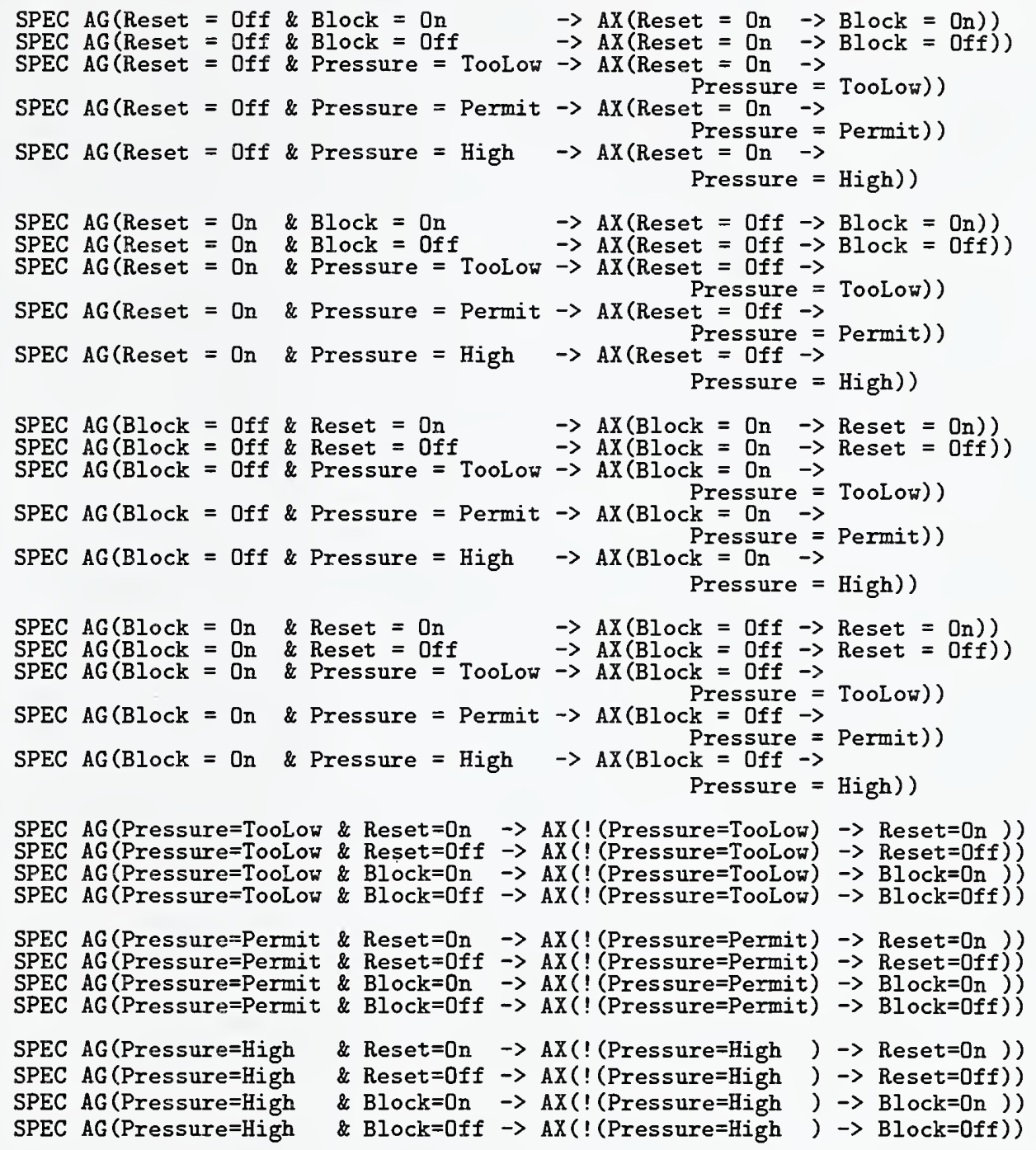




